\title{
Measurement of non-equilibrium carriers dynamics in Ce-doped YAG, LuAG and GAGG crystals with and without Mg-codoping
}

\author{
M.T. Lucchini ${ }^{a 1}$, O. Buganov ${ }^{b}$, E. Auffray ${ }^{a}$, P. Bohacek ${ }^{c}$, M. Korjik ${ }^{d}$, D. Kozlov ${ }^{d}$, S. Nargelas ${ }^{e}$, M. Nikl ${ }^{c}$,

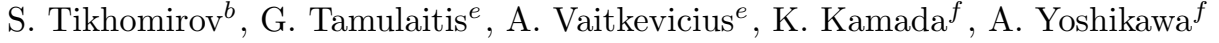 \\ ${ }^{a}$ CERN, Geneva, Switzerland \\ ${ }^{b}$ Stepanov Institute of Physics, Minsk, Belarus \\ ${ }^{c}$ Institute of Physics AS CR, Prague, Czech Republic \\ ${ }^{d}$ Research Institute for Nuclear Problems, Minsk, Belarus \\ ${ }^{e}$ Vilnius University, Vilnius, Lithuania \\ ${ }^{f}$ CESA Corporation, T-Biz, Sendai, Japan
}

\begin{abstract}
Non-linear absorption spectroscopy in pump and probe configuration has been used to test the population of nonequilibrium carriers in Ce-doped $\mathrm{Y}_{3} \mathrm{Al}_{5} \mathrm{O}_{12}$ (YAG), $\mathrm{Lu}_{3} \mathrm{Al}_{5} \mathrm{O}_{12}$ (LuAG), and $\mathrm{Gd}_{3} \mathrm{Al}_{x} \mathrm{Ga}_{(5-x)} \mathrm{O}_{12}$ (GAGG) crystals with and without codoping by $\mathrm{Mg}^{2+}$ ions. A faster rise time of the induced optical density has been observed in all crystals codoped with $\mathrm{Mg}$ with respect to that in Mg-free samples. A significant difference in the time evolution of the differential optical density in GAGG with respect to YAG and LuAG crystals has also been measured. In both GAGG:Ce and GAGG:Ce,Mg an absorption band with maximum in the blue-green range and a decay time of $1.4 \mathrm{ps}$ is present. This band is due to the absorption by free electrons before they are trapped or re-captured by $\mathrm{Ce}^{3+}$ ions. A broad absorption band in the yellow-red region with very short rise time and a decay time longer than 150 ps has been observed in all the Ce-doped garnets under study and can be attributed to the absorption from the $\mathrm{Ce}^{3+}$ excited states.
\end{abstract}

Keywords: Scintillators, Multi-component garnet crystals, Non-linear absorption spectroscopy, Excitation transfer

\section{Introduction}

The rise time of the scintillation signal plays a crucial role in defining the time resolution achievable with a given scintillator, which depends on the interplay between the 5 light yield and the kinetics of the scintillation process [1. A shorter and brighter scintillation pulse leads to a smaller jitter on the arrival time of the first photons at the photodetector. Therefore, these parameters are crucial in defining the potential application of inorganic scintillators for 10 both time of flight positron emission tomography (TOFPET) scans 2, 3, 4, and precision timing detectors in high energy physics experiments [5].

Recently, Ce-doped garnets with the chemical formula $\mathrm{M}_{3} \mathrm{~A}_{5} \mathrm{O}_{12}$, in which the metal $\mathrm{M}$ can be either a single 15 yttrium / rare earth ion or their mixture; and the metal A can be Al, Ga or their mixture, have been extensively studied. Their main characteristics are high light yield, scintillation decay time shorter than $100 \mathrm{~ns}$, and emission peaks around $540 \mathrm{~nm}$, which can be exploited with the

\footnotetext{
${ }^{1}$ Corresponding author

Postal address: CERN, 1211 Geneva 23 (Switzerland)

E-mail address: Marco.Toliman.Lucchini@cern.ch
}

20 good sensitivity of silicon photo-multipliers (SiPM) at such wavelength.

The codoping of Ce-doped scintillation materials with alkali earth ions has been recently demonstrated to be a promising approach to improve the scintillation properties, especially in the scintillators with a garnet-type crystal matrix [6, 7. Such improvement is a combined result of the modification of electronic excitation transfer processes, achieved by band gap engineering, and the tailoring of the activator level energy within the gap, implemented via a variation of the garnet composition [8, 9]. The codoping of the crystal using alkali earth ions results in the substitution of a trivalent ion in the host matrix with a divalent second-group cation and causes the formation of anionic vacancies that compensate the charge deficiency. The formation of a hole-type defect including $\mathrm{Mg}^{2+}$ and $\mathrm{O}^{-}$in close proximity of the divalent cation is also favored [10, 11. Moreover, the codoping of Ceactivated crystals by divalent ions (even at the level of a few hundreds ppm) causes the oxidation of a fraction of the $\mathrm{Ce}^{3+}$ ions into $\mathrm{Ce}^{4+}$. Both these types of cerium ions are then involved in the scintillation process but with different kinetics [12, 13, 14]. The result is a significant accelera- 
tion of the luminescence rise and decay times, as observed in $\mathrm{Gd}_{3} \mathrm{Al}_{2.3} \mathrm{Ga}_{2.7} \mathrm{O}_{12}$ (GAGG:Ce) crystals codoped with $45 \mathrm{Mg}$ [15]. This leads, as expected, to an improvement of the coincidence time resolution, as recently demonstrated for $\mathrm{GAGG}, \mathrm{Y}_{3} \mathrm{Al}_{5} \mathrm{O}_{12}$ : $\mathrm{Ce}$ (YAG:Ce), and $\mathrm{Lu}_{3} \mathrm{Al}_{5} \mathrm{O}_{12}$ : $\mathrm{Ce}$ (LuAG:Ce) crystals [16, 17].

With respect to YAG and LuAG crystals, GAGG shows 50 a peculiar feature: it contains numerous levels of ${ }^{7} \mathrm{f}$ electronic states in the band gap, due to the presence of $\mathrm{Gd}^{3+}$ ions. As recently shown in [18, the ground state ${ }^{8} \mathrm{~S}$ of $\mathrm{Gd}^{3+}$ ions in GAGG crystals is located about $1 \mathrm{eV}$ below the top of the valence band. A substantial difference in 55 the excitation transfer to the radiating level of $\mathrm{Ce}^{3+}$ in GAGG is thus expected with respect to Gd-free garnets. Since the luminescence build-up process is determined by the population of the $\mathrm{Ce}^{3+}$ radiating levels, studying the time evolution of the population is a powerful tool to inves-

60 tigate both the luminescence response and the dynamics of free carriers before they are captured by traps and ra-100 diative centers. To study these processes, the population of the lowest excited levels can be probed by exploiting the optical pump-and-probe technique in which a femtosecond 65 pump pulse is used for photo-excitation and a probe pulse of similar duration but with variable delay (with respect to105 the excitation pulse) is used to probe the free carrier density and the population of the excited electronic energy levels. In the current paper, a comparison of the fast ex70 citation transfer processes in Ce-doped GAGG, YAG, and LuAG crystals, with and without Mg-codoping, is pro-110 vided.

\section{Experimental Methods}

The six Ce-doped garnet samples used in this study115 ${ }_{75}$ were grown by Czochralski technique at a speed of about 1 $\mathrm{mm}$ /hour using an iridium crucible under $\mathrm{N}_{2}$ atmosphere, or, in case of GAGG, under atmosphere of $\mathrm{N}_{2}$ with $2 \%$ of $\mathrm{O}_{2}$. For the GAGG samples, the seed crystal of $\langle 100\rangle$ orientation was purchased from C\&A Corporation, Sendai,120

80 Japan. Mixtures of oxides of purity $5 \mathrm{~N}$ with compositions of $\mathrm{Gd}_{2.982} \mathrm{Ce}_{0.015} \mathrm{Ga}_{2.7} \mathrm{Al}_{2.3} \mathrm{O}_{12}$ and $\mathrm{Gd}_{2.982} \mathrm{Ce}_{0.015} \mathrm{Mg}_{0.003}$ $\mathrm{Ga}_{2.7} \mathrm{Al}_{2.3} \mathrm{O}_{12}$ were used as starting materials. For the YAG and LuAG samples a stoichiometric mixture of $4 \mathrm{~N}$ $\mathrm{MgCO}_{3}, \mathrm{CeO}_{2}$, a- $\mathrm{Al}_{2} \mathrm{O}_{3}, \mathrm{Y}_{2} \mathrm{O}_{3}$ and $\mathrm{Lu}_{2} \mathrm{O}_{3}$ powders was 85 used as starting material. Nominally, starting powders were prepared according to the formula of $\left(\mathrm{Mg}_{0.005} \mathrm{Ce}_{0.0005125}\right.$ $\left.\mathrm{Lu}_{0.99}\right)_{3} \mathrm{Al}_{5} \mathrm{O}_{12}$ and $\left(\mathrm{Mg}_{0.005} \mathrm{Ce}_{0.0005} \mathrm{Y}_{0.99}\right)_{3} \mathrm{Al}_{5} \mathrm{O}_{12}$ and seeds of $\langle 111\rangle$ oriented YAG crystals were used. More details on the production method and the chemical compositions are

90 described elsewhere [16, 17. All the samples were cut from single crystal ingots in the shape of a $2 \times 2 \times 10 \mathrm{~mm}^{3}$ pix-130 els and subsequently polished. The standard scintillation and optical properties of these samples were measured and discussed in [17.

The study of non-equilibrium carrier dynamics was performed using a pump-probe spectrometer based on an orig-135 inal femtosecond Ti: $\mathrm{Al}_{2} \mathrm{O}_{3}$ pulsed oscillator and a regen-

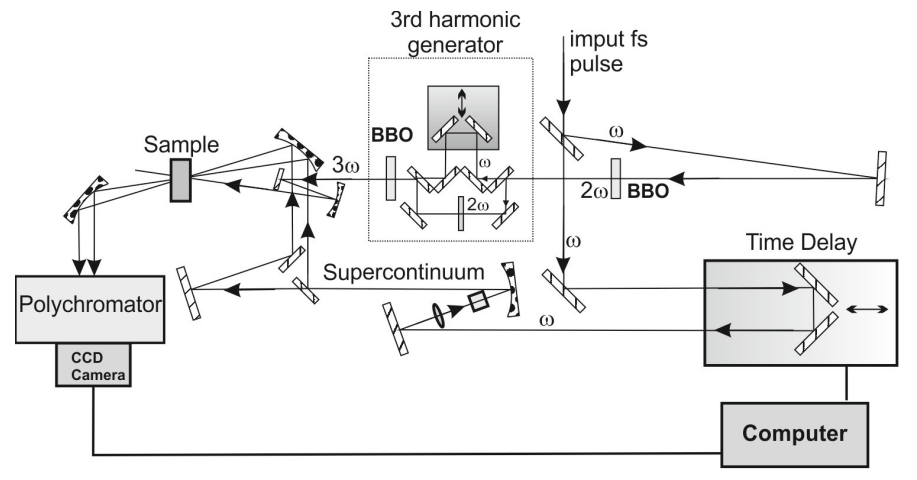

Figure 1: Schematic diagram of the pump-probe spectrometer setup used to study non-equilibrium carrier dynamics.

erative amplifier, both operating at $10 \mathrm{~Hz}$ repetition rate. A schematic diagram of the setup is shown in Fig. 1. The laser pulse duration and energy after amplification were 140 fs and up to $0.5 \mathrm{~mJ}$, respectively, while the wavelength was tunable over the spectral range from 770 to $820 \mathrm{~nm}$. The pulses of the fundamental frequency (the wavelength, $\lambda$, was set at $790 \mathrm{~nm}$ for the present study) were divided after the output of the amplifier into two parts at a ratio of 1:4. The beam of higher intensity was converted to the third harmonic $(\lambda \approx 263 \mathrm{~nm}$, pulse energy $\mathrm{E}$ up to 12 $\mu \mathrm{J})$ and used as the pump pulse. The pulse energy was selected low enough to prevent the sample surface from optical damage by the laser pulse. The second beam of smaller intensity was used as a probe.

After passing the delay line, the probe pulse was converted to a white super-continuum generated by focusing the beam into a $1 \mathrm{~cm}$ long water cell. By using a semitransparent mirror, the super continuum radiation (360$800 \mathrm{~nm}$ ) was subdivided into two pulses (reference and signal) of similar intensity. Both pulses were focused on the sample by mirror optics. The reference pulse was exploited to eliminate the impact of shot-to-shot instability of super-continuum. It passes the sample always before the pump pulse. The pump-induced change of the optical density was calculated as:

$$
\Delta D(\lambda, \Delta t)=\log \left[\frac{E_{s g}(\lambda)}{E_{r e f}(\lambda)} \cdot \frac{E_{r e f}(\lambda, \Delta t)}{E_{s g}^{*}(\lambda, \Delta t)}\right]
$$

where $E_{s g}, E_{s g}^{*}$, and $E_{r e f}$ are the energies of the signal pulses which passed the sample before and after the pump pulse, and the reference pulse, respectively. The beam spot diameter was $0.5 \mathrm{~mm}$. The spectra of both pulses were recorded for each laser shot by a system including a polychromator equipped with a CCD camera and digitally processed. The absorption spectra of the white super continuum were measured in the spectral range from 400 to $700 \mathrm{~nm}$. All measurements have been performed at room temperature. Additional details on the setup are provided in [19, 20, 21, 22], where the same technique and instrumental apparatus have been used for studies of ultrafast phenomena in different materials. 

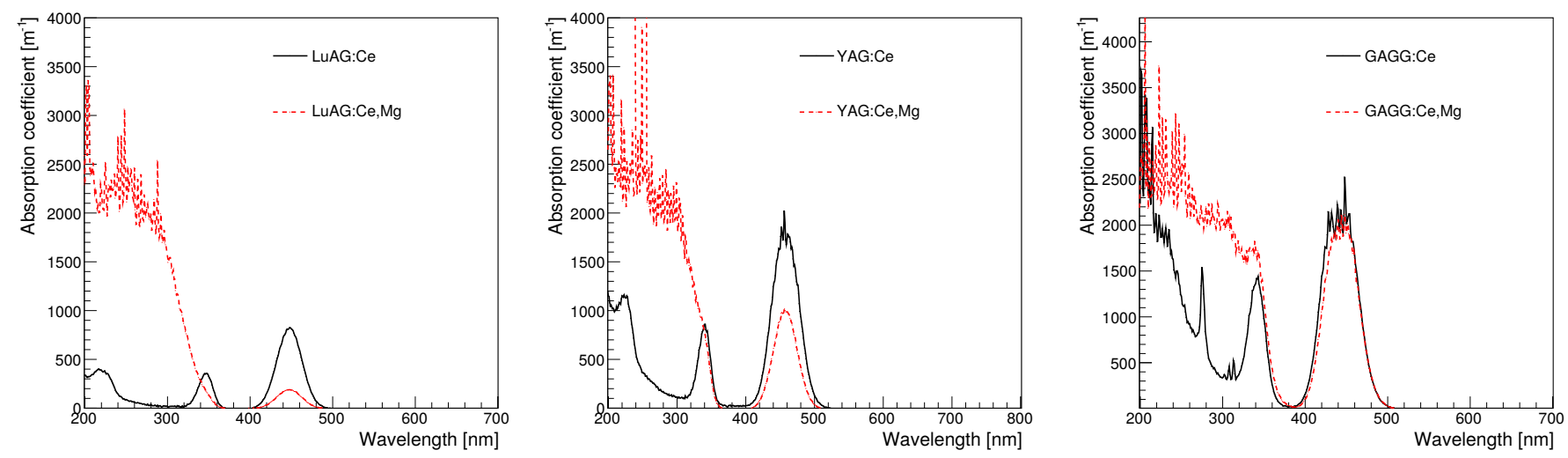

Figure 2: Absorption spectra of LuAG:Ce, YAG:Ce and GAGG:Ce without (black solid) and with Mg codoping (red dotted). Fluctuations at low wavelengths are due to the lower light beam intensity of the spectrophotometer and larger light absorption by the crystals in the UV spectral range. These effects deteriorate the signal-to-noise ratio of the measurement.

\section{Results}

The absorption spectra of the crystals under study, measured through $2 \mathrm{~mm}$ thickness using a Perkin Elmer VVIS) spectrometer, are shown in Fig. 2 All the Mg-free crystals exhibit the $4 \mathrm{f}-5 \mathrm{~d}$ absorption bands corresponding to inter-configuration transitions of $\mathrm{Ce}^{3+}{ }^{180}$ ions. The samples codoped with $\mathrm{Mg}^{2+}$ ions additionally show a broad absorption band in the UV region (below 300 . This band, related to the charge transfer (CT) transition from the valence band to a defect stabilized by $\mathrm{Mg}^{2+}$ (e.g. $\mathrm{Ce}^{4+}$ ions) 10 , is more pronounced in the LuAG:Ce crystals having the lowest concentration of $\mathrm{Ce}^{3+}$ ions. The absorption spectrum of the GAGG:Ce sample also shows set of narrow absorption bands in the UV range corresponding to the transitions from ${ }^{8} \mathrm{~S}$ ground state to the numerous components of $\mathrm{P}, \mathrm{I}$, and $\mathrm{D}$ terms split by spinorbit interaction.

The transient induced absorption spectra of all samples

155 for different delay times between the pump and the probe pulses are shown in Fig. 3. The pump wavelength, $\lambda_{e x}=$ $263 \mathrm{~nm}$, was chosen to avoid direct excitation of $\mathrm{Gd}^{3+{ }^{19}}$ states in GAGG crystals. For all the samples under study, such wavelength excites the $\mathrm{Ce}^{3+}$ ions in the tail of the third component of the inter-configuration transition $4 \mathrm{f}^{1}$ $5 \mathrm{~d}^{1} 4 \mathrm{f}^{0}[23$. Since the corresponding excited level is located in the conduction band (CB), the de-localization of the non-equilibrium electrons and their capture might easily occur. In addition, the pump causes CT transitions in the defect centers due to the divalent ions introduced by the Mg-codoping. The differential optical density spectra of GAGG:Ce and YAG:Ce show a drop around 440-450 $\mathrm{nm}$, in correspondence with the first $4 \mathrm{f}^{1}-5 \mathrm{~d}^{1} 4 \mathrm{f}^{0}$ allowed transition of $\mathrm{Ce}^{3+}$ ions. This occurs due to a relatively larger concentration of $\mathrm{Ce}^{3+}$ in these samples with respect to the lower dopant concentration in LuAG.

The spectra of GAGG:Ce samples contain two overlapping and wide absorption bands (local maxima in the spectrum): a first band, B1, having its maximum in the ${ }^{210}$ peaking around $680 \mathrm{~nm}$. As shown in the differential absorption kinetics, in Fig. 4. these bands appear simultaneously with the leading edge of the excitation. The B1 band (probed at $480 \mathrm{~nm}$ ) shows an extremely fast decay component with time constant of $\tau_{d, 1}=1.4 \pm 0.2 \mathrm{ps}$ followed by another component with slower decay constant ( $\sim 150 \mathrm{ps})$. The time evolution of the B2 band intensity (probed at $680 \mathrm{~nm}$ ) shows no fast component and decays with a comparably slow time constant as B1.

The B1 band is considerably less resolved in the absorption spectra of YAG:Ce and LuAG:Ce samples and no fast decay component in the kinetics is observed for the differential optical density probed at a spectral wavelength close to the B1 band (around $400 \mathrm{~nm}$ ). The B2 band appears to have its maximum around $650 \mathrm{~nm}$ in YAG and LuAG samples and its slow decay time constant is also longer than in GAGG:Ce samples: it is about $400 \mathrm{ps}$ for Mg-codoped LuAG and YAG and larger than 1000 ps for the respective $\mathrm{Mg}$-free samples.

A comparison of the initial part of the differential optical density kinetics in LuAG:Ce, YAG:Ce and GAGG:Ce, with and without Mg-codoping, is better shown within a reduced time range in Fig. 5. The time evolution of the transient absorption in the Mg-codoped GAGG:Ce is similar to that in the sample without codoping, except for the fast component B1 which becomes faster and of smaller intensity. A faster rise time is also visible for LuAG:Ce and YAG:Ce Mg-codoped crystals with respect to the respective Mg-free samples and is further discussed in the following section.

\section{Discussion}

There is a significant difference between the defect formation process in YAG, LuAG and GAGG crystals. In garnet crystals containing two or more cations and isovalent doping ions, e.g. $\mathrm{Ce}^{3+}$ in our case, the defects are cre- 
LuAG:Ce $-\lambda_{\text {ex }}=263 \mathrm{~nm}$

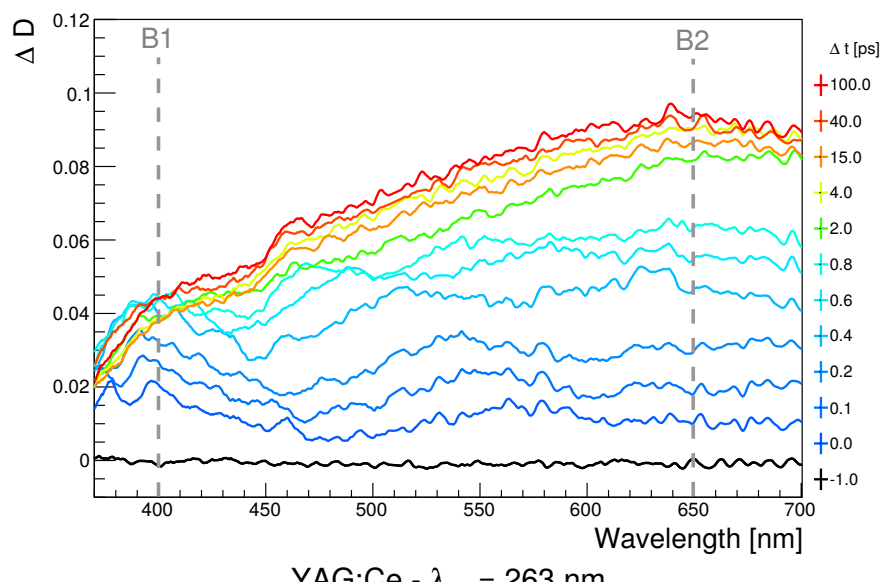

YAG:Ce $-\lambda_{\text {ex }}=263 \mathrm{~nm}$

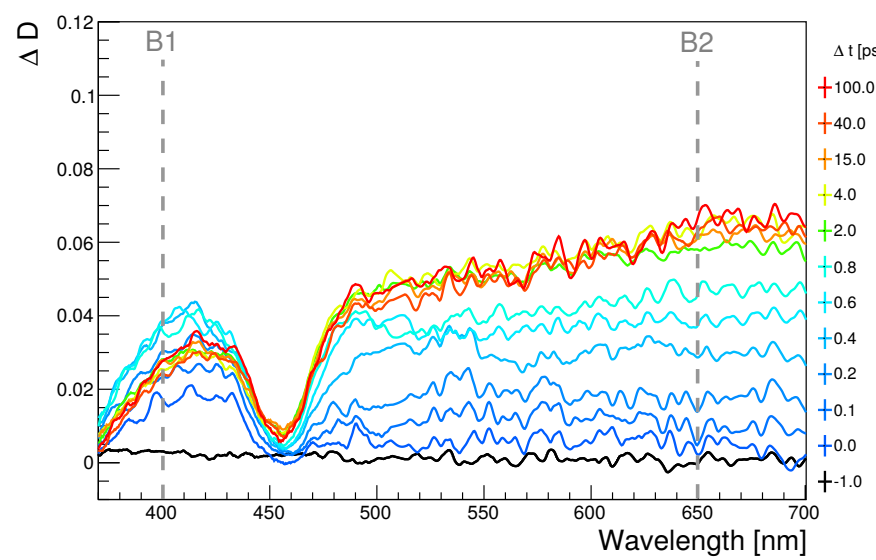

GAGG:Ce $-\lambda_{\text {ex }}=263 \mathrm{~nm}$

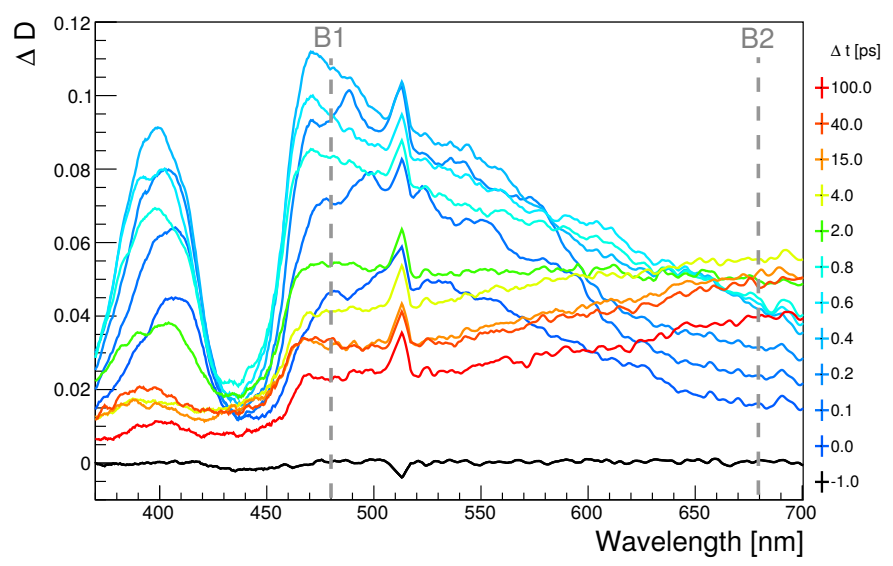

LuAG:Ce,Mg $-\lambda_{\text {ex }}=263 \mathrm{~nm}$

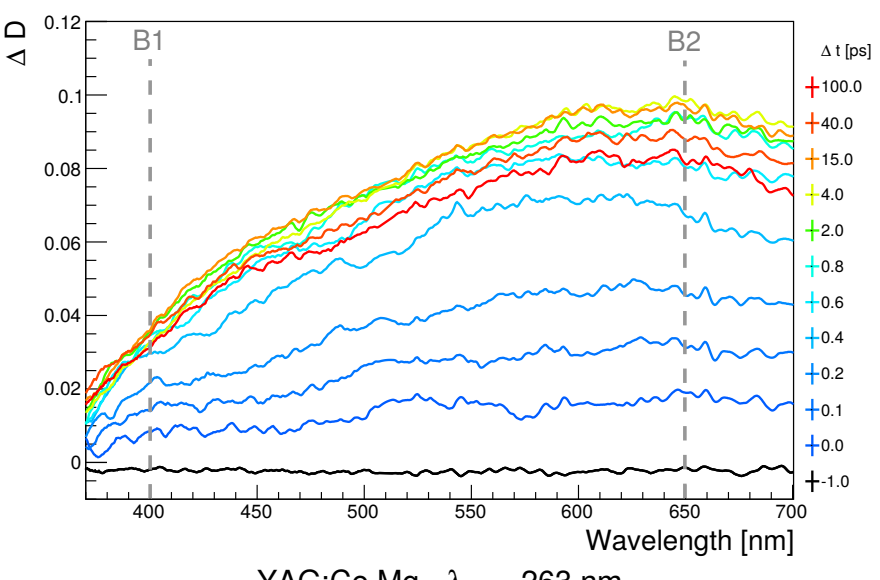

YAG:Ce,Mg $-\lambda_{\text {ex }}=263 \mathrm{~nm}$

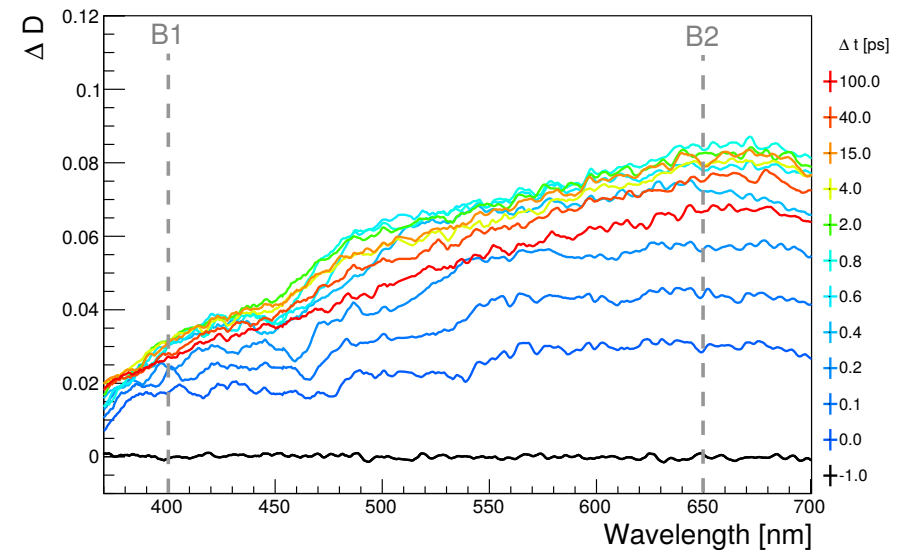

GAGG:Ce,Mg $-\lambda_{\mathrm{ex}}=263 \mathrm{~nm}$

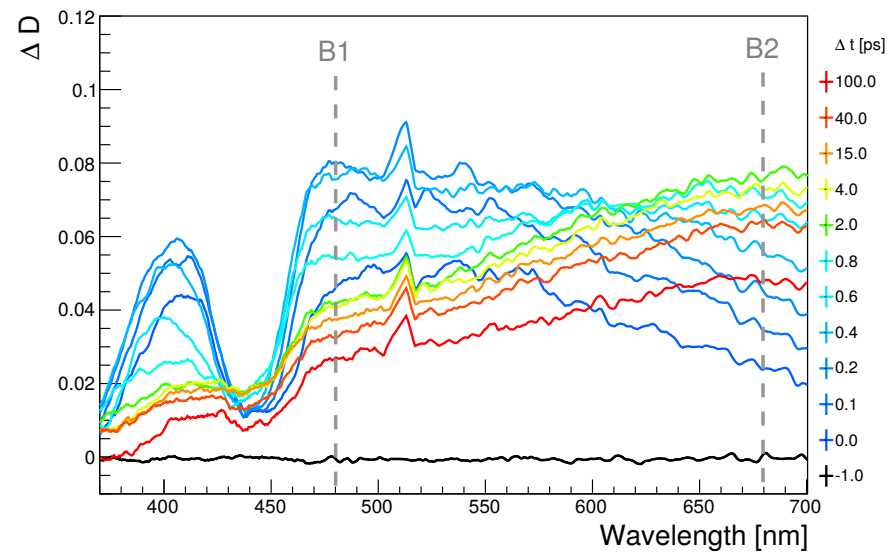

Figure 3: Transient induced absorption spectra of LuAG:Ce, YAG:Ce and GAGG:Ce single crystals (from top to bottom) with (left) and without (right) Mg-codoping. The spectra are shown at different time delays between the pump and probe pulses (indicated) at pump pulse energy of $10 \mu \mathrm{J}$.

ated by the preferential evaporation of the most volatile component from the melt during the crystal growth. In $_{220}$ this case, randomly distributed vacancies will dominate. In GAGG crystals, where gallium is the most volatile component, the formation of cation vacancies due to the gallium evaporation inevitably leads to the formation of anionic vacancies and, consequently, to a higher concentra-225 tion of trapping centers with respect to garnets grown from binary compositions. For example, oxygen vacancies create a set of electron trap levels with activation energy in the range up to $1 \mathrm{eV}$. Moreover, multicomponent garnet crystals favor the formation of additional defects that act as trapping centers for non-equilibrium carriers. The ratio of the ionic radii of $\mathrm{Al}$ and $\mathrm{Ga}$ is $0.39 \AA$ to $0.47 \AA$ in the oxygen tetrahedral positions and $0.53 \AA$ to $0.62 \AA$ in octahedral positions [24]. Therefore, even a random dis- 
LuAG:Ce $-\lambda_{\text {ex }}=263 \mathrm{~nm}$
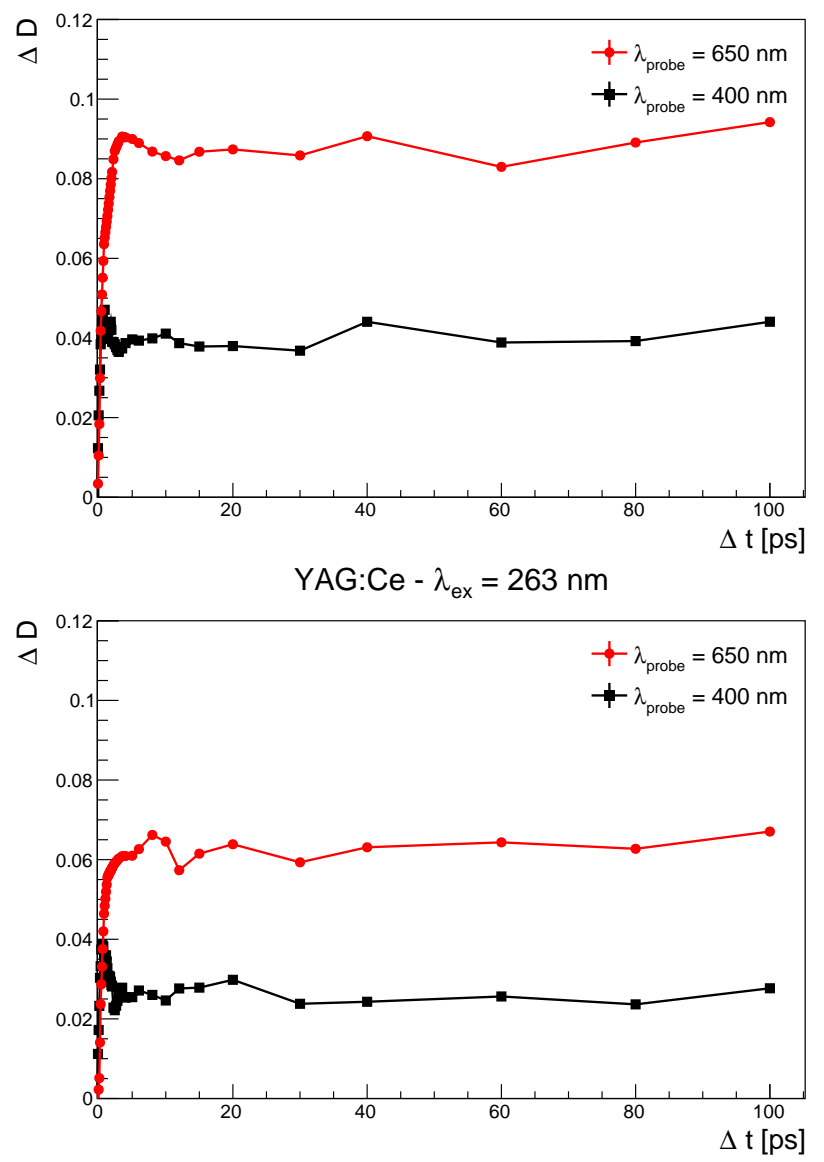

GAGG:Ce $-\lambda_{\mathrm{ex}}=263 \mathrm{~nm}$

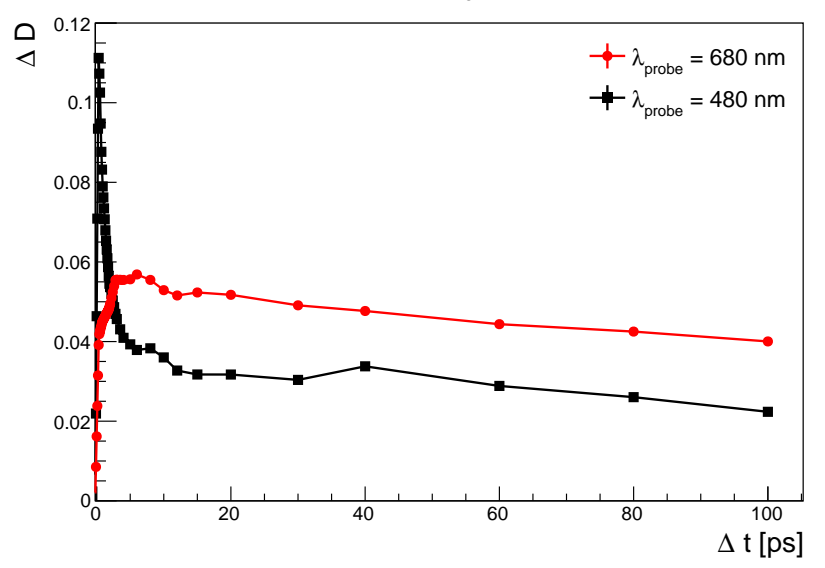

LuAG:Ce,Mg $-\lambda_{\mathrm{ex}}=263 \mathrm{~nm}$
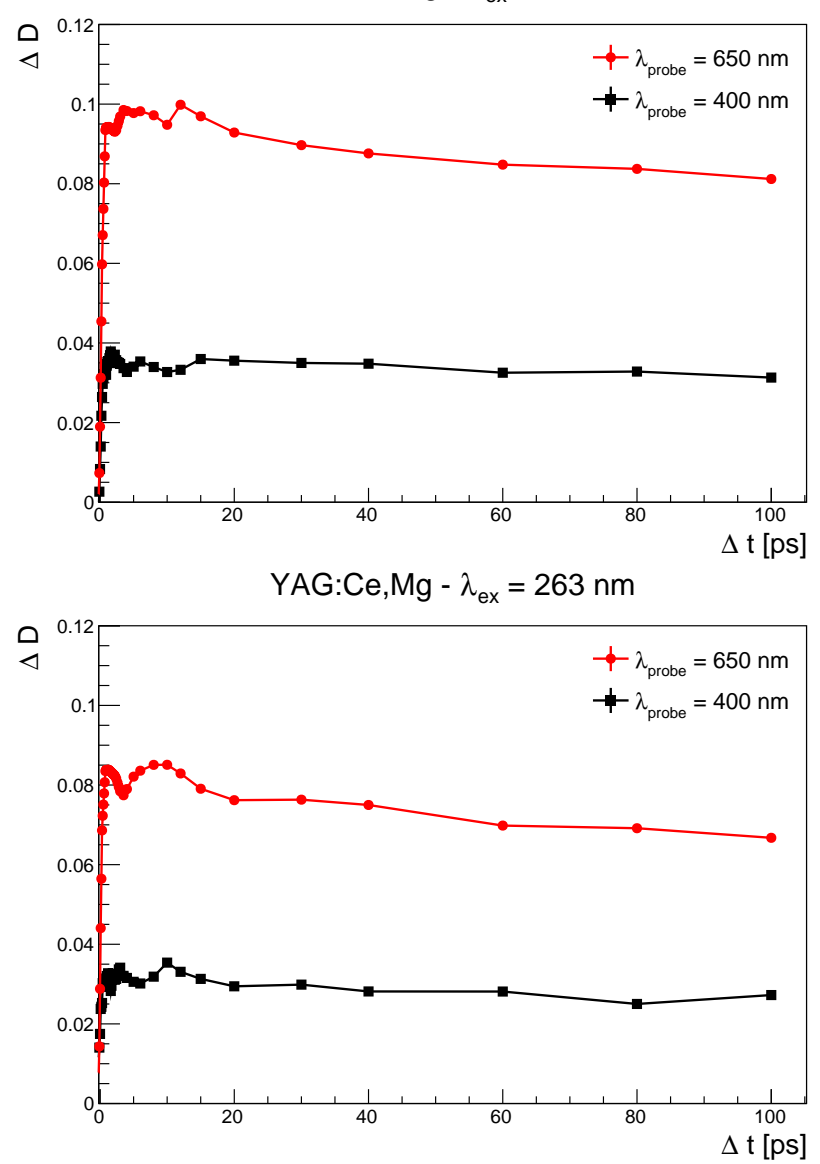

GAGG:Ce,Mg $-\lambda_{\text {ex }}=263 \mathrm{~nm}$

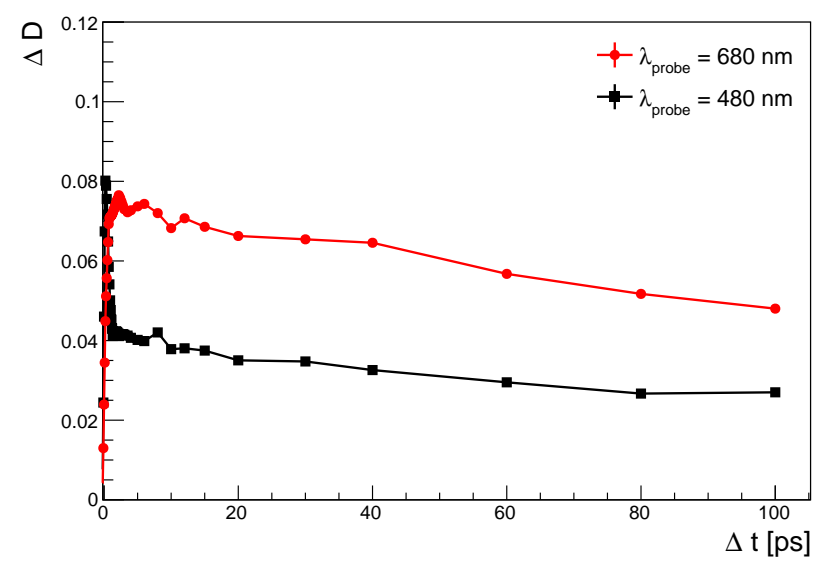

Figure 4: Time evolution of the probe differential absorption, $\Delta D$, at two different spectral regions of 400-480 and 650-680 nm for LuAG:Ce, YAG:Ce and GAGG:Ce single crystals (from top to bottom) with (left) and without (right) Mg-codoping.

tribution of $\mathrm{Al}$ and $\mathrm{Ga}$ ions in the lattice results in considerable distortion of the lattice. Closely located gallium and aluminum ions distort the polyhedral environment of other and result in the formation of additional shallow trapping centers. For this reason, a higher concentra-240 tion of shallow centers is expected in GAGG with respect to YAG and LuAG crystals. It is in fact reflected in low temperature TSL glow curve of these materials [25, 26]. sient absorption of GAGG:Ce samples was previously observed in [27. The results presented in this work confirm its presence in GAGG:Ce and show that such component is absent in YAG:Ce and LuAG:Ce crystals, independently from the presence of $\mathrm{Mg}^{2+}$ ions. This observation suggests that the transient absorption band peaking in the bluegreen region, B1, cannot be associated with the excited radiating level of $\mathrm{Ce}^{3+}$. Most probably, the $\mathrm{B} 1$ band is caused by free electron absorption, where the free electrons 

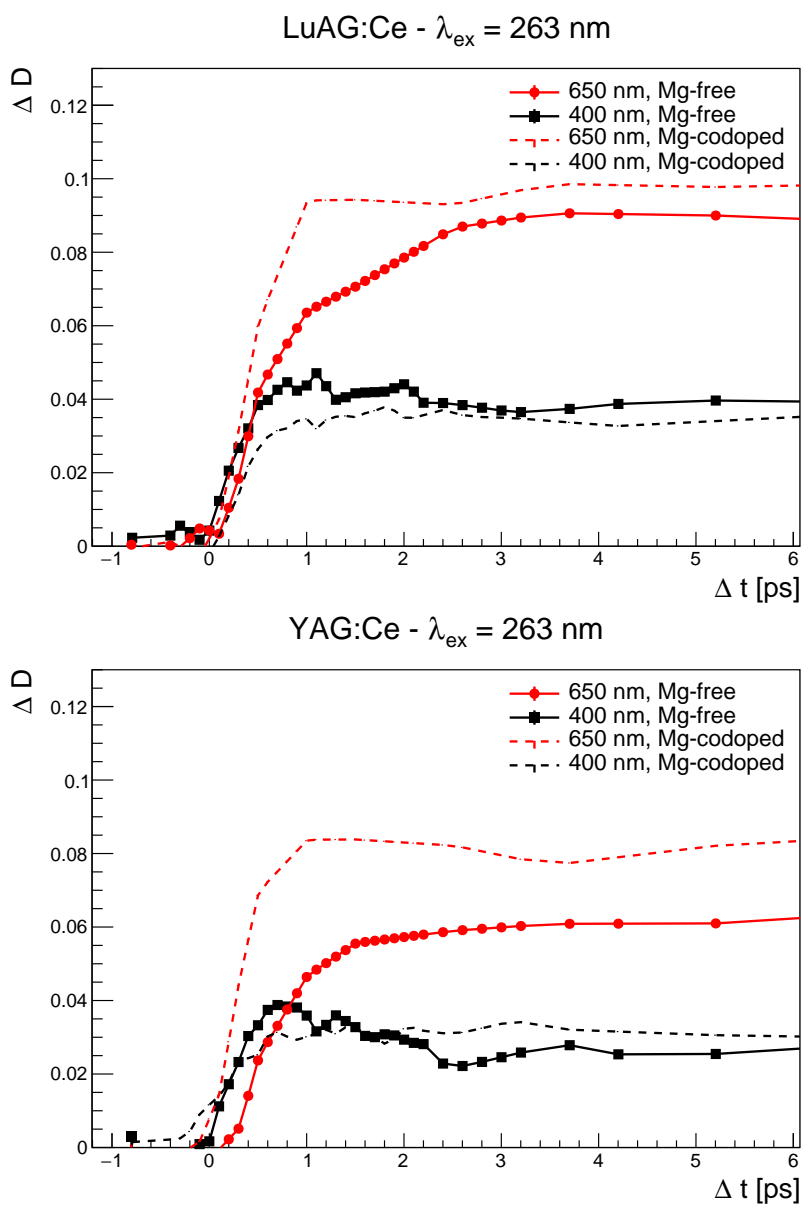

GAGG:Ce $-\lambda_{\text {ex }}=263 \mathrm{~nm}$

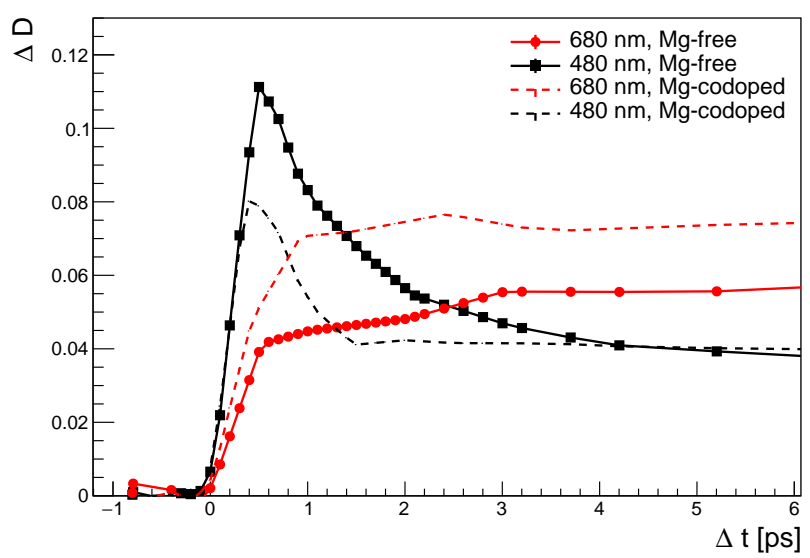

Figure 5: Comparison of the early kinetics of the probe differential absorption, $\Delta D$, at two different spectral regions of 400-480 and $650{ }^{29}$ $680 \mathrm{~nm}$ between $\mathrm{Mg}$-codoped (dotted line) and $\mathrm{Mg}$-free (solid line) samples of LuAG:Ce, YAG:Ce and GAGG:Ce single crystals (from top to bottom). are generated via the absorption by $\mathrm{Ce}^{3+}$ and the subsequent transfer of the electrons to the conduction band. The spectral width of the B1 band might be attributed to structured density of states in the conduction band 28.

The fast decay of the free electron absorption can be explained by the localization of free electrons at the traps ${ }_{305}$ in GAGG. Conversely, a maximum of the transient ab- sorption around 650-680 nm (broad B2 band) is observed in all samples under study and could be attributed to the absorption from the $\mathrm{Ce}^{3+}$ excited radiating levels.

This interpretation is also supported by the different kinetic behavior of the B1 and B2 absorption bands observed in the GAGG:Ce sample at the initial stage after the short-pulse excitation. The equilibrium between the free electrons and the electrons at the excited $\mathrm{Ce}^{3+}$ level is established within approximately 2 ps after the pump excitation, while the further decay of both free electron density (probed at $480 \mathrm{~nm}$ ) and the population of the excited $\mathrm{Ce}^{3+}$ level (probed at $680 \mathrm{~nm}$ ) proceeds at a similar rate, which is determined by radiative and non-radiative recombinamagnesium, the relative contribution of the fast component is smaller and its decay time faster $\left(\tau_{d}=0.5 \pm 0.04\right.$ ps) indicating that an additional recombination channel might have been introduced by codoping.

A difference in the rising time of the transient B2 absorption band kinetics between Mg-codoped and Mg-free garnets has also been observed as shown in Fig. 5. This difference is minimal in GAGG crystal but, in YAG and LuAG samples, the rising edge of the transient absorpthe Mo-codoped crystals is considerably shorter due to capturing of free electrons from shallow traps. The $\mathrm{Mg}^{2+}$-based defect centers, having a CT transition peaked at $265 \mathrm{~nm}$, create deep trap levels approximately $1.5 \mathrm{eV}$ below the bottom of conduction band. This can cause the same electrons to be captured multiple times from shallow traps and prevents them from being re-captured by $\mathrm{Ce}^{3+}$ ions via the conduction band. Shallow traps capture electrons from the conduction band, while the thermal re-activation of the electrons back to the conduction band results in the population of $\mathrm{Ce}^{3+}$ radiating levels and is responsible for the delayed luminescence observed in such samples. In Mg-codoped crystals, this process is substantially suppressed by the re-capturing of trapped electrons by Mg-related centers and thus the re-population from the decay is faster in the codoped crystals. Meanwhile, the additional channel of non-radiative recombination, which is introduced by Mg-codoping, reduces the scintillation efficiency of garnet crystals.

\section{Conclusions}

The transient differential absorption of a set of Cedoped $\mathrm{Y}_{3} \mathrm{Al}_{5} \mathrm{O}_{12}, \mathrm{Lu}_{3} \mathrm{Al}_{5} \mathrm{O}_{12}$ and $\mathrm{Gd}_{3} \mathrm{Al}_{2} \mathrm{Ga}_{3} \mathrm{O}_{12}$ crystals with and without Mg-codoping has been measured by pumpprobe method using femtosecond laser sources. The pump at the wavelength of $263 \mathrm{~nm}$ was used to create free carriers in all the samples under study, whereas white supercontinuum was applied to measure transient absorption in the spectral range from 400 to $700 \mathrm{~nm}$.

Substantial differences in the dynamics of population of the excited state of the activator ions in three garnet-type 
scintillators: YAG:Ce, LuAG:Ce, and GAGG:Ce were revealed. Two non-linear absorption bands were observed. The first band, present in all three garnets, is broad and extends from approximately $450 \mathrm{~nm}$ over the whole visible region and decays with a characteristic time constant $\gtrsim 150$ ps. This band is attributed to the absorption from the lowest excited state of $\mathrm{Ce}^{3+}$. The second band is peaked at the blue-green region, emerges in the sub-picosecond domain and has a decay time of $\sim 1.4 \mathrm{ps}$. The time evolution of this band implies that it is caused by the light absorption by free electrons released to the conduction $_{375}$ band after photo-excitation of $\mathrm{Ce}^{3+}$ ions and rapidly captured by trapping states. This band is most pronounced in GAGG:Ce due to the influence of the second excited state of $\mathrm{Ce}^{3+}$, which is in the conduction band close to its ${ }_{380}$ bottom, and is hardly traced in LuAG:Ce.

Pairs of samples of the crystals, nominally identical except of codoping by magnesium, were prepared to reveal the influence of codoping by divalent ions. We show that ${ }_{385}$ Mg-codoping introduces additional levels into the band gap, which facilitate transferring the trapped electrons back to the Ce ions.

\section{Acknowledgments}

This work was performed in the framework of the Crystal Clear Collaboration and received funding from the Eu-395 ropean Union's Horizon 2020 research and innovation program under the Marie Skodowska-Curie grant agreement no. 644260 (Intelum). Support has been received also from the AIDA2020 European project, the ASCIMAT project 400 under grant agreement no.690599 and from COST Action TD1401 (FAST). We also acknowledge the support from the Belarus Foundation for Fundamental Research and by the Czech Science Foundation 16-15569S.

\section{References}

[1] S. Gundacker, et al., Measurement of intrinsic rise times for ${ }^{410}$ various $\mathrm{L}(\mathrm{Y}) \mathrm{SO}$ and $\mathrm{LuAG}$ scintillators with a general study of prompt photons to achieve 10 ps in TOF-PET, Phys. Med. Biol. 61(7) (2016) 2802-37. doi:10.1088/0031-9155/61/7/2802

[2] W. W. Moses, et al., Time of flight in PET revisited, IEEE Trans. Nucl. Sci. 50 (2003) 1325-30. doi:10.1109/TNS.2003. ${ }^{15}$ 817319

[3] M. Conti, et al., State of the art and challenges of time-of-flight PET, Phys. Med. 25 (2009) 1-1. doi:10.1016/j.ejmp. 2008. 10.001

350 [4] S. Gundacker, et al., State of the art timing in TOF-PET de- ${ }^{420}$ tectors with $\mathrm{LuAG}, \mathrm{GAGG}$ and $\mathrm{L}(\mathrm{Y}) \mathrm{SO}$ scintillators of various sizes coupled to FBK-SiPMs, Journal of Instrumentation 11 (08) (2016) P08008

[5] CMS Collaboration, Technical proposal for the Phase-II upgrade of the Compact Muon Solenoid, CERN-LHCC-2015-010 (2015) LHCC-P-008.

[6] K. Kamada, T. Yanagida, J. Pejchal, M. Nikl, T. Endo, K. Tsutumi, Y. Fujimoto, A. Fukabori, A. Yoshikawa, Scintillatororiented combinatorial search in Ce-doped $(\mathrm{Y}, \mathrm{Gd})_{3}(\mathrm{Ga}, \mathrm{Al})_{5} \mathrm{O}_{12}$ multicomponent garnet compounds, Journal of Physics D: Ap- ${ }^{430}$ plied Physics 44 (50) (2011) 505104.
[7] K. Kamada, T. Yanagida, J. Pejchal, M. Nikl, T. Endo, K. Tsutsumi, Y. Fujimoto, A. Fukabori, A. Yoshikawa, Crystal growth and scintillation properties of Ce-doped $\mathrm{Gd}_{3}(\mathrm{Ga}, \mathrm{Al})_{5} \mathrm{O}_{12}$ single crystals, IEEE Transactions on Nuclear Science 59 (5) (2012) 2112-2115. doi:10.1109/TNS.2012.2197024

[8] I. I. Vrubel, R. G. Polozkov, I. A. Shelykh, V. M. Khanin, P. A. Rodnyi, C. R. Ronda, Bandgap engineering in YttriumAluminum Garnet with Ga-doping, Crystal Growth \& Design 17 (4) (2017) 1863-1869. doi:10.1021/acs.cgd.6b01822.

[9] M. Fasoli, A. Vedda, M. Nikl, C. Jiang, B. P. Uberuaga, D. A. Andersson, K. J. McClellan, C. R. Stanek, Band-gap engineering for removing shallow traps in rare-earth $\mathrm{Lu}_{3} \mathrm{Al}_{5} \mathrm{O}_{12}$ garnet scintillators using $\mathrm{Ga}^{3+}$ doping, Phys. Rev. B 84 (2011) 081102. doi:10.1103/PhysRevB.84.081102

[10] M. Nikl, V. Babin, J. Pejchal, V. V. Laguta, M. Buryi, J. A. Mares, K. Kamada, S. Kurosawa, A. Yoshikawa, D. Panek, T. Parkman, P. Bruza, K. Mann, M. Mller, The stable $\mathrm{Ce}^{4+}$ Center: A new tool to optimize Ce-doped oxide scintillators, IEEE Transactions on Nuclear Science 63 (2) (2016) 433-438. doi:10.1109/TNS.2015.2495119.

[11] C. Hu, S.-P. Liu, M. Fasoli, A. Vedda, M. Nikl, X.-Q. Feng, Yu-Bai, ESR and TSL study of hole and electron traps in LuAG:Ce,Mg ceramic scintillator, Optical Materials 45 (2015) 252 - 257. doi:10.1016/j.optmat.2015.03.049

[12] S. Blahuta, et al., Evidence and consequences of $\mathrm{Ce}^{4+}$ in LYSO:Ce,Ca and LYSO:Ce,Mg single crystals for medical imaging applications, IEEE Trans. Nucl. Sci. 60 (2013) 3134-3141. doi:10.1088/0031-9155/60/12/4635

[13] Y. Wu, et al., Role of $\mathrm{Ce}^{4+}$ in the scintillation mechanism of co-doped $\mathrm{Gd}_{3} \mathrm{Al}_{2} \mathrm{Ga}_{3} \mathrm{O}_{12}$ :Ce, Phys. Rev. Applied 241 (2014) 044009. doi:10.1103/PhysRevApplied.2.044009

[14] M. Nikl, et al., Defect engineering in Ce-doped aluminum garnet single crystal scintillators, Cryst. Growth Des. 14 (2014) 48274833. doi:10.1021/cg501005s

[15] E. Auffray, R. Augulis, A. Borisevich, V. Gulbinas, A. Fedorov, M. Korjik, M. Lucchini, V. Mechinsky, S. Nargelas, E. Songaila, G. Tamulaitis, A. Vaitkeviius, S. Zazubovich, Luminescence rise time in self-activated $\mathrm{PbWO}_{4}$ and Ce-doped $\mathrm{Gd}_{3} \mathrm{Al}_{2} \mathrm{Ga}_{3} \mathrm{O}_{12}$ scintillation crystals, Journal of Luminescence 178 (2016) $54-$ 60. doi:10.1016/j.jlumin.2016.05.015

[16] M. Lucchini, V. Babin, P. Bohacek, S. Gundacker, K. Kamada, M. Nikl, A. Petrosyan, A. Yoshikawa, E. Auffray, Effect of $\mathrm{Mg}^{2+}$ ions co-doping on timing performance and radiation tolerance of Cerium doped $\mathrm{Gd}_{3} \mathrm{Al}_{2} \mathrm{Ga}_{3} \mathrm{O}_{12}$ crystals, Nucl. Instrum. Meth. A816 (2016) 176 - 183. doi:10.1016/j.nima.2016.02.004

[17] M. Lucchini, S. Gundacker, P. Lecoq, A. Benaglia, M. Nikl, K. Kamada, A. Yoshikawa, E. Auffray, Timing capabilities of garnet crystals for detection of high energy charged particles, Nuclear Instruments and Methods in Physics Research Section A: Accelerators, Spectrometers, Detectors and Associated Equipment 852 (2017) 1-9. doi:10.1016/j.nima.2017.02.008.

[18] E. Auffray, M. Korjik, M. Lucchini, S. Nargelas, O. Sidletskiy, G. Tamulaitis, Y. Tratsiak, A. Vaitkeviius, Free carrier absorption in self-activated $\mathrm{PbWO}_{4}$ and Ce-doped $\mathrm{Y}_{3}\left(\mathrm{Al}_{0.25} \mathrm{Ga}_{0.75}\right)_{3} \mathrm{O}_{12}$ and $\mathrm{Gd}_{3} \mathrm{Al}_{2} \mathrm{Ga}_{3} \mathrm{O}_{12}$ garnet scintillators, Optical Materials 58 (2016) 461 - 465. doi:10.1016/j.optmat. 2016.06.040

[19] A. J. Taylor, E. S. Davies, J. A. Weinstein, I. V. Sazanovich, O. V. Bouganov, S. A. Tikhomirov, M. Towrie, J. McMaster, C. D. Garner, Ultrafast intramolecular charge separation in a donoracceptor assembly comprising bis(5cyclopentadienyl)molybdenum coordinated to an ene-1,2dithiolate-naphthalenetetracarboxylicdiimide ligand, Inorganic Chemistry 51 (24) (2012) 13181-13194. doi:10.1021/ ic301436t

[20] V. I. Stsiapura, A. A. Maskevich, S. A. Tikhomirov, O. V. Buganov, Charge transfer process determines ultrafast excited state deactivation of thioflavin $\mathrm{t}$ in low-viscosity solvents, The Journal of Physical Chemistry A 114 (32) (2010) 8345-8350. doi:10.1021/jp105186z

[21] O. V. Buganov, A. S. Grabtchikov, Y. I. Malakhov, Y. M. 
Popov, V. A. Orlovich, S. A. Tikhomirov, Features of raman amplification in $\mathrm{kgw}$ and barium nitrate crystals at excitation 435 by femtosecond pulses Laser Physics Letters 9 (11) (2012) 786 . URL http://stacks . iop. org/1612-202X/9/i=11/a=005

[22] M. Smirnov, O. Buganov, E. Shabunya-Klyachkovskaya, S. Tikhomirov, O. Ovchinnikov, A. Vitukhnovsky, A. Perepelitsa, A. Matsukovich, A. Katsaba, Dynamics of electronic excitations relaxation in hydrophilic colloidal CdS quantum dots in gelatin with involvement of localized states, Physica E: Lowdimensional Systems and Nanostructures 84 (Supplement C) (2016) 511 - 518. doi:10.1016/j.physe.2016.07.004

[23] P. A. Tanner, L. Fu, L. Ning, B.-M. Cheng, M. G. Brik, Soft synthesis and vacuum ultraviolet spectra of yag:ce 3+ nanocrystals: reassignment of ce $3+$ energy levels, Journal of Physics: Condensed Matter 19 (21) (2007) 216213.

[24] R. D. Shannon, Revised effective ionic radii and systematic studies of interatomic distances in halides and chalcogenides, 450 Acta Crystallographica Section A 32 (5) (1976) 751-767. doi: 10.1107/S0567739476001551.

[25] K. Brylew, W. Drozdowski, A. J. Wojtowicz, K. Kamada, A. Yoshikawa, Studies of low temperature thermoluminescence of GAGG:Ce and LuAG:Pr scintillator crystals using the $\mathrm{T}_{\text {max }}{ }^{-}$ $\mathrm{T}_{\text {stop }}$ method, Journal of Luminescence 154 (2014) $452-457$. doi:10.1016/j.jlumin.2014.05.035

[26] W. Drozdowski, K. Brylew, M. Witkowski, A. Wojtowicz, P. Solarz, K. Kamada, A. Yoshikawa, Studies of light yield as a function of temperature and low temperature thermolumines460 cence of $\mathrm{Gd}_{3} \mathrm{Al}_{2} \mathrm{Ga}_{3} \mathrm{O}_{12}$ :Ce scintillator crystals, Optical Man terials 36 (10) (2014) 1665 - 1669, sI: IWASOM'13. doi: 10.1016/j.optmat.2013.12.044.

[27] G. Tamulaitis, A. Vaitkeviius, S. Nargelas, R. Augulis, V. Gulbinas, P. Bohacek, M. Nikl, A. Borisevich, A. Fedorov, M. Korjik, E. Auffray, Scintillator-oriented combinatorial search in Ce-doped $(\mathrm{Y}, \mathrm{Gd})_{3}(\mathrm{Ga}, \mathrm{Al})_{5} \mathrm{O}_{12}$ multicomponent garnet compounds, Nucl. Instruments Methods Phys. Res. Sect. A Accel. Spectrometers, Detect. Assoc. Equip. (2017) (to be published)

[28] Y.-N. Xu, W. Y. Ching, Electronic structure of yttrium aluminum garnet $\left(\mathrm{Y}_{3} \mathrm{Al}_{5} \mathrm{O}_{12}\right)$, Phys. Rev. B 59 (1999) 1053010535. doi:10.1103/PhysRevB.59.10530 